\title{
Structural changes in the intervertebral disc
}

\author{
MALCOLM I. V. JAYSON*† AND JENNIFER S. BARKS $\dagger$ \\ From the Department of Medicine, University of Bristol*, and the Royal National Hospital for \\ Rheumatic Diseases, Bath $\dagger$
}

Back pain is an extremely common symptom and is experienced by over half the adult population (Lawrence, 1969). In some cases, well-defined causes such as a prolapsed intervertebral disc or ankylosing spondylitis can be recognized, but in the majority no precise cause can be identified. In this paper, a series of studies of the structure of the intervertebral disc is reported, and possible mechanisms for the production of spinal symptoms are suggested.

\section{Specimens and methods}

Spines were removed from 33 cadavers at the end of autopsy examinations. The specimens were removed intact from the first lumbar to the first sacral vertebra avoiding damage to the intervertebral discs. They were $x$ rayed and stored at $-20^{\circ} \mathrm{C}$. Only spines which were radiologically normal or which showed minimal degenerative changes were used. Thirteen spines were rejected on account of more severe $x$ ray changes. The ages of the subjects from whom the remaining twenty spines were obtained varied between 18 and 77 years. Each spine was sliced horizontally into individual segments containing one disc and the centre of each disc was injected from the anterior surface with a radio-opaque water-soluble dye (Conray 480) sometimes mixed with Evans Blue. By this technique, injury to the posterior fibres of the annulus fibrosus was avoided. Each disc was separately $x$ rayed, not only in the lateral and antero-posterior directions, but also in the axial or vertical direction, a view which cannot be obtained during life. The specimens were re-frozen in liquid nitrogen to $-196^{\circ} \mathrm{C}$. and then sliced using a band saw in the saggital, coronal, or horizontal planes, in order to demonstrate further the structure of the annulus fibrosus and nucleus pulposus.

An attempt was made to correlate the experimental findings with the clinical history of spinal problems. This usually proved impossible, as details regarding back pain were not usually recorded in patients admitted with fatal illnesses. Only limited information could be obtained from the patients' practitioners.

\section{Results}

The normal intervertebral disc consists of a central nucleus pulposus surrounded by the annulus fibrosus. These fibres pursue a course around the circumference of the disc and spiral up and down from one vertebra to those above and below. Abnormalities in disc structure, which were common, particularly in the older spines, were classified into various types:

Accepted for publication June 14, 1972.
(1) Antero-posterior position of the nucleus pulposus The lateral discograms and the saggital sections of the intervertebral discs demonstrated the anteroposterior position of the nucleus pulposus. Typically, in the upper lumbar discs, the nucleus pulposus is central (Fig. 1). A thick band of fibres of the annulus :fibrosus runs up and down both in front and behind

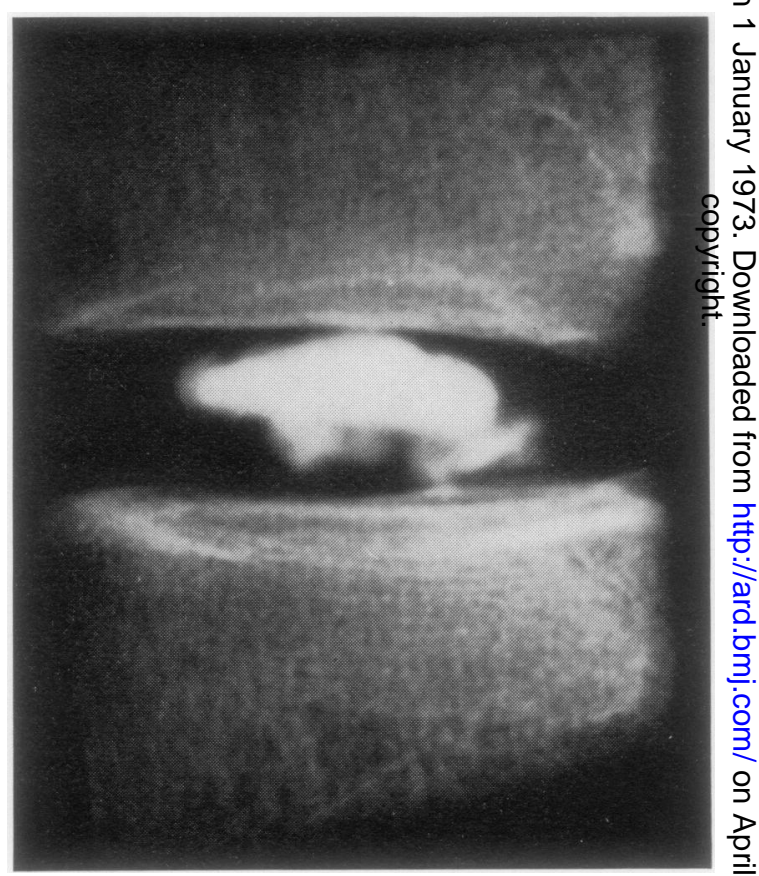

FIG. 1 Lateral discogram of $L_{23}$ disc, showing central م position of nucleus pulposus

the nucleus. However, in the lower lumbar vertebrae, the nucleus is often placed more posteriorly. This is usually (but not invariably) most marked in the L5/S1 disc, where the posterior edge of the nucleus often reaches the posterior border of the vertebrae $\stackrel{?}{+}$ (Fig. 2). A very thick band of annulus fibrosus fibres lie anteriorly, but, posteriorly, the fibre band is thin and pursues a hairpin bend behind the nucleus to pass from one vertebral body to the other. These $\stackrel{\mathbb{Q}}{\varrho}$ fibres produce a posterior bulge and press on the $\bar{\sigma}$ posterior longitudinal ligament (Fig. 3). 


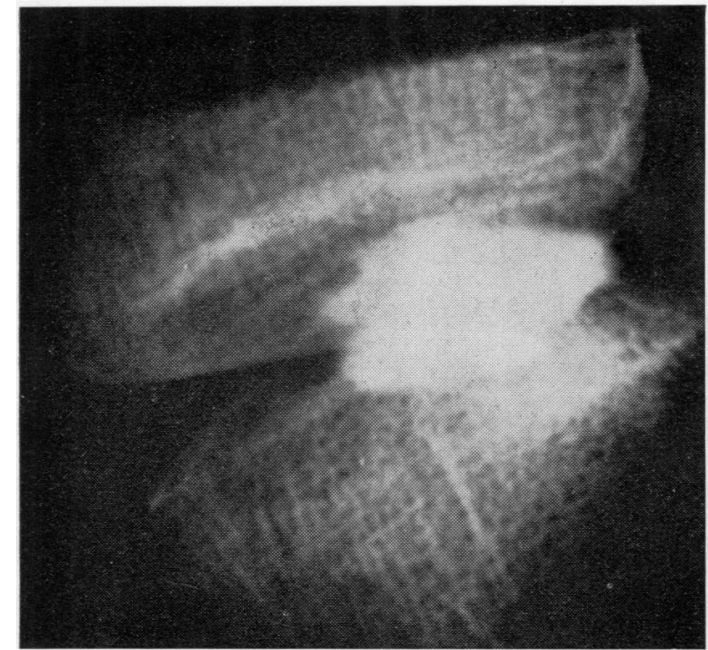

FIG. 2 Lateral discogram of $L_{5} S_{1}$, showing posterior position of nucleus pulposus

(2) Postero-lateral extension of the nucleus pulposus In intervertebral discs which otherwise appear completely normal, there is a tendency for the nucleus to extend in the postero-lateral direction on both sides. This was demonstrated both in axial discograms
(Fig. 4) and by horizontal section of the disc. When this type of postero-lateral extension is present, it often occurs in several discs of the same spine.

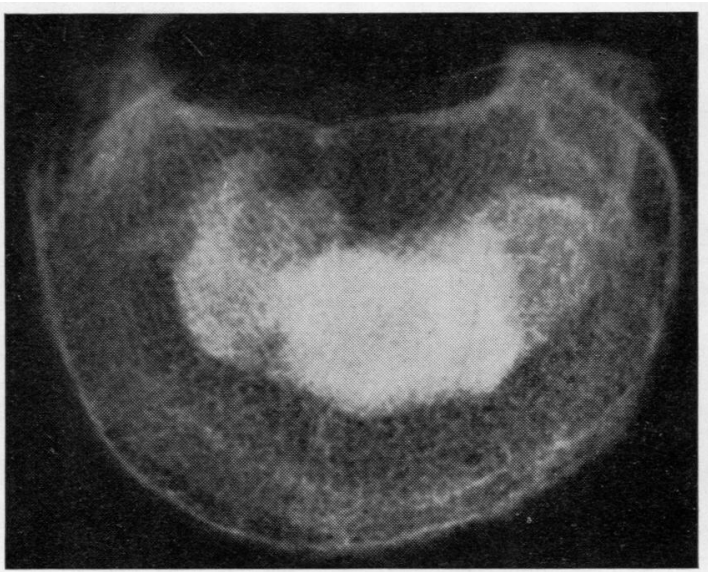

FIG. 4 Axial discogram, showing postero-lateral extensions of nucleus pulposus

\section{(3) Central posterior herniation}

In a number of specimens both the axial discogram and the horizontal section of the disc demonstrated

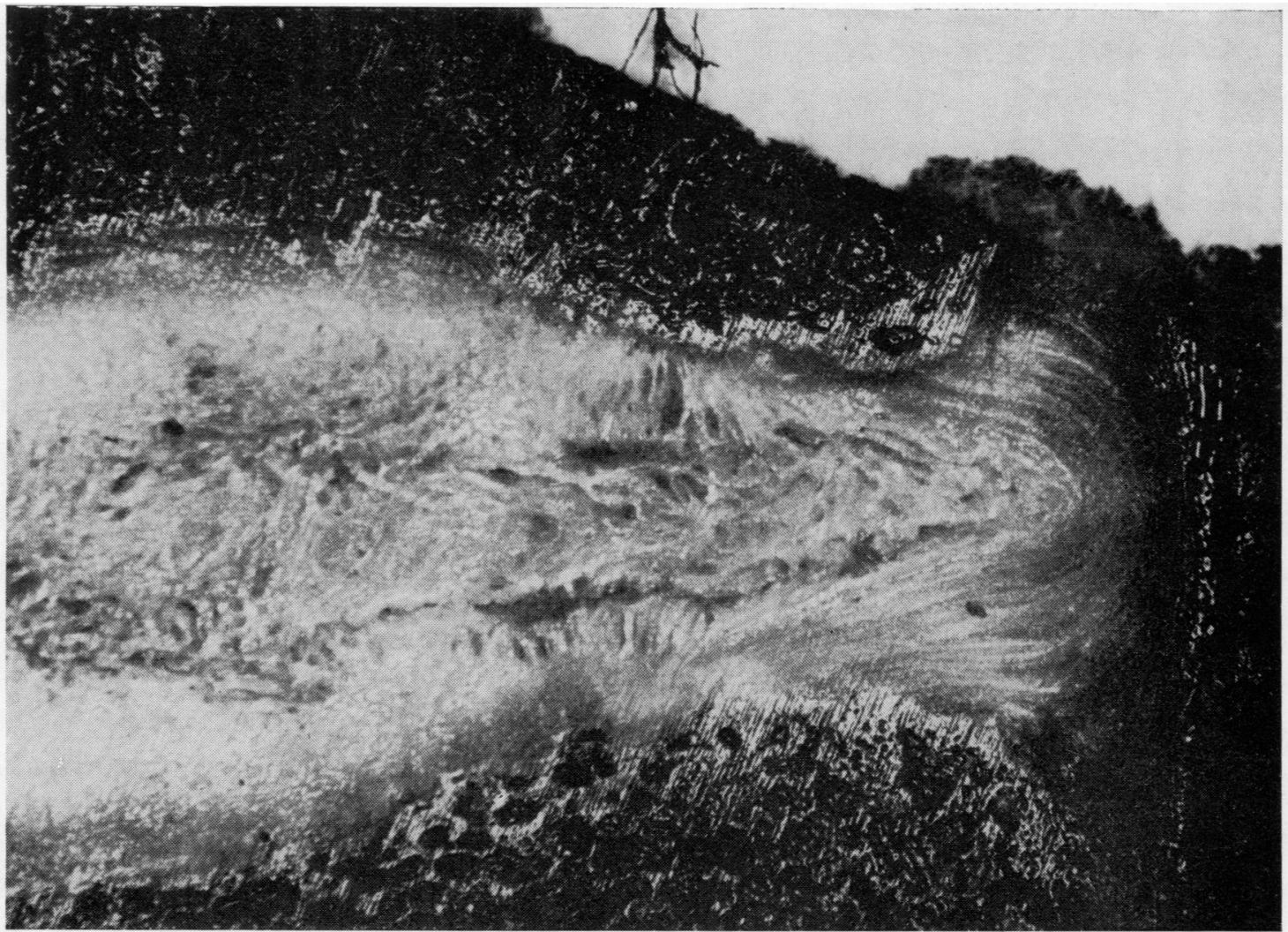

FIG. 3 Sagittal section of posterior half of $L_{5} S_{1}$ disc, showing hairpin bend and posterior bulge of annulus fibrosus 

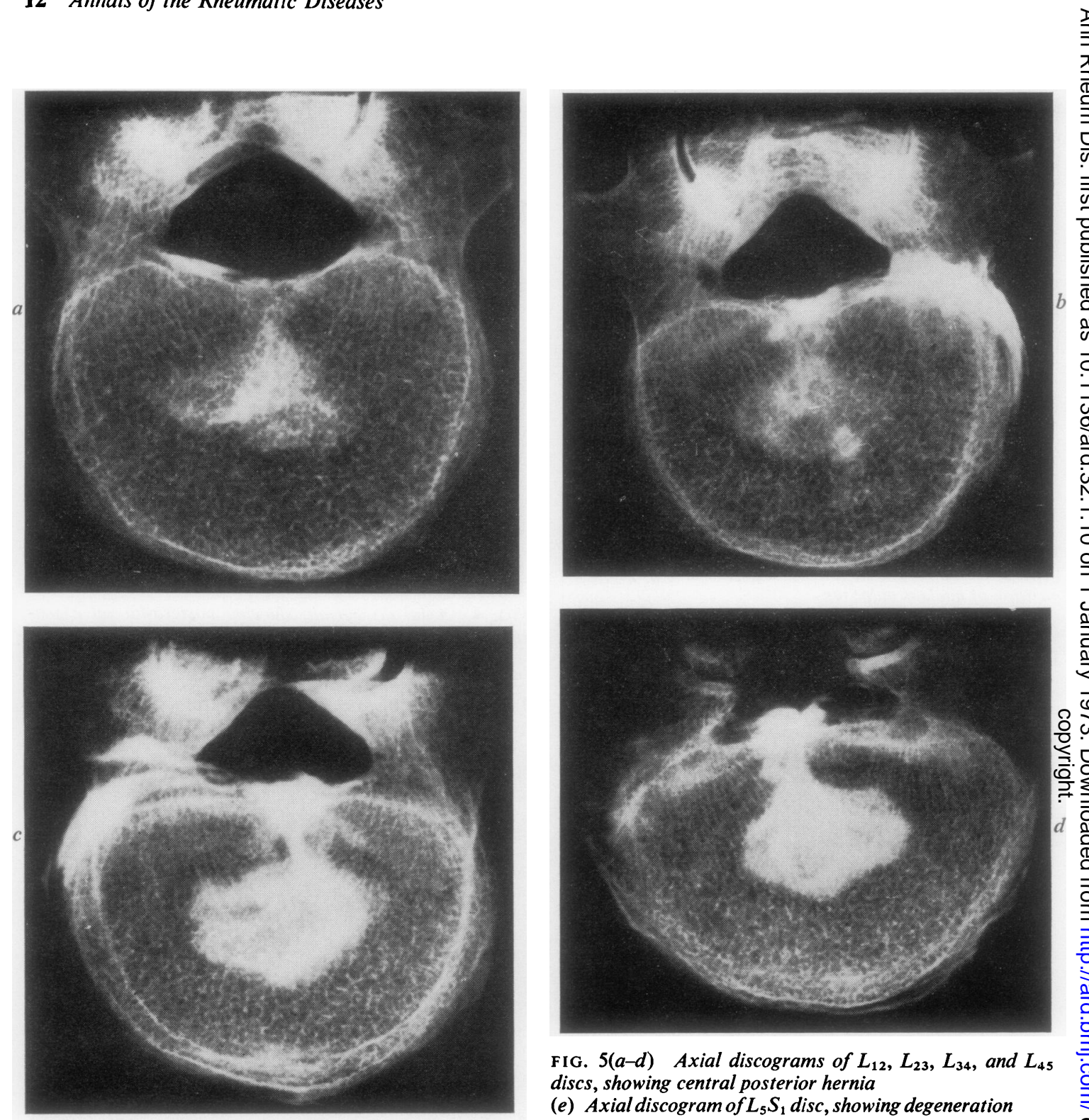

FIG. 5(a-d) Axial discograms of $L_{12}, L_{23}, L_{34}$, and $L_{45}$ discs, showing central posterior hernia

(e) Axial discogram of $L_{5} S_{1}$ disc, showing degeneration

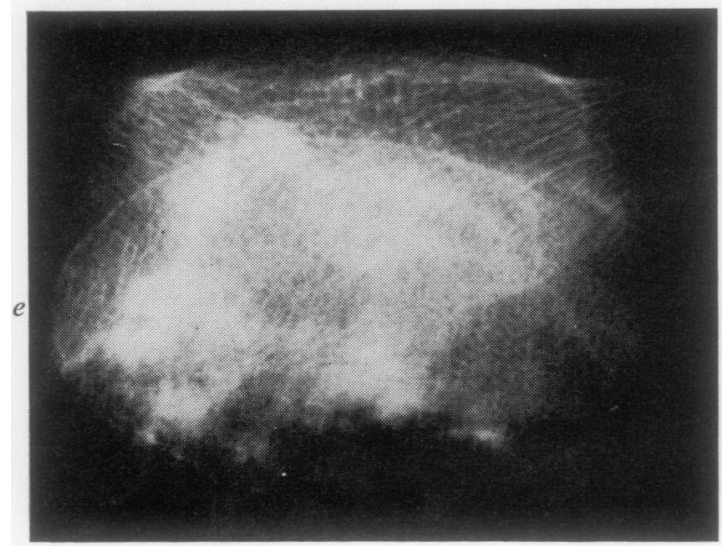

nuclear material herniating posteriorly through a narrow neck to lie just beneath the posterior longitudinal ligament. Again when this occurred it was often found in multiple discs of the same spine. Fig. 5a-d shows such an example occurring in the $\mathrm{L} 1 / 2, \mathrm{~L} 2 / 3, \mathrm{~L} 3 / 4$, and $\mathrm{L} 4 / 5$ discs, with degeneration of the $\mathrm{L}_{5} \mathrm{~S}_{1}$ disc (Fig. 5e) in a patient who had suffered recurrent back pain for many years, but with a completely normal spinal $x$ ray. He had been classified as 'neurotic'. The hernia passed through a narrow neck and fibres could be seen orientated around the neck of the prolapse (Fig. 6). The appearance of the $\mathrm{L}_{5} \mathrm{~S}_{1}$ disc in horizontal section suggested that degeneration had followed this type of disc damage (Fig. 7, overleaf). 


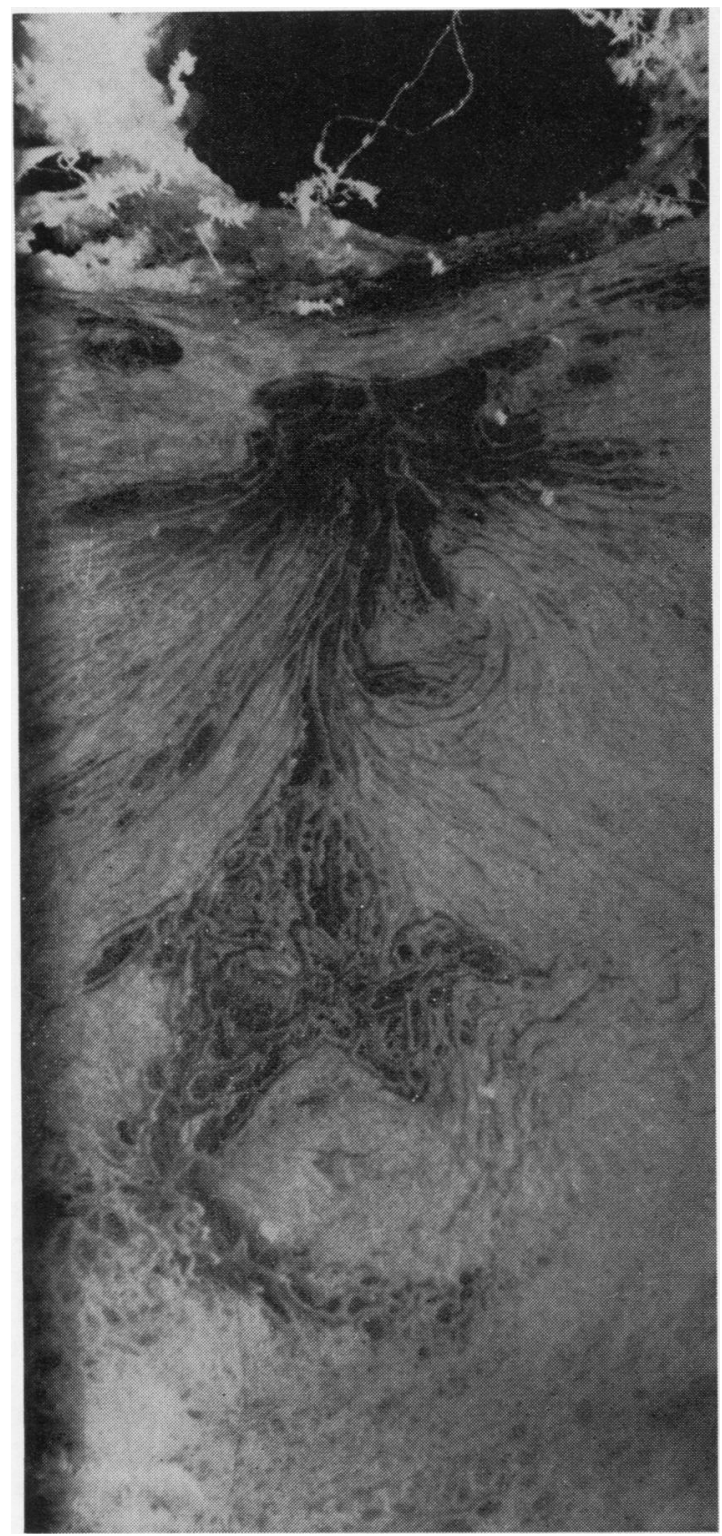

FIG. $6 L_{23}$ disc. Horizontal section, showing path taken by herniated nuclear material.

\section{(4) Postero-lateral prolapse}

Herniation of the disc substance through a posterolateral prolapse was frequently observed in the axial discograms and horizontal section (Fig. 8). When present, this again was frequently found at several disc levels in the same spine. Examination of the neck of the prolapse revealed disturbance of the fibres in the direction taken by the nuclear material.

\section{Discussion}

Recurrent back pain is an extremely common problem, and yet the mechanisms involved in its produc- tion have not been elucidated. Pain fibres are to be found in the spinal ligaments, in the capsules of the apophyseal joints, in the periosteum-particularly at fascial and tendon attachments, and in the walls of blood vessels, and these structures may all be relevant to spinal symptoms. It is generally agreed that disorders of the invertebral discs must play a large part in the production of spinal pain. Pain fibres are present in the intervertebral discs of the foetus but they degenerate by birth. There are no nerve endings within the intervertebral disc in adults (Roofe, 1940; Stilwell, 1956; Hirsch, Ingelmark, and Miller, 1963; Jackson, Winkelmann, and Bickel, 1966; Wyke, 1970). The nerve supply to the area behind the disc is provided by the sinu-vertebral nerve (Pedersen, Blunck, and Gardner, 1956), which originates from the spinal nerve just distal to the ganglion and reenters the spinal canal to reach the midline. It gives branches directly towards the discs above and below each level. The fibres supply the posterior longitudinal ligament, the periosteum, the blood vessels, and the dura. Roofe (1940), Stilwell (1956), Hirsch and others (1963), Jackson and others (1966), and Wyke (1970) were able to demonstrate fine unmyelinated nerve fibres at the attachment of the posterior longitudinal ligament to the back of the annulus fibrosus, but none was found within the annulus fibrosus. Clearly, if pain is arising because of disorders of the intervertebral disc, it must be generated at the posterior edge of the annulus fibrosus where it fuses with the posterior longitudinal ligament.

Anatomical studies of the position of the normal nucleus pulposus show that it often occupies a more posterior position in the lower lumbar discs. In the upper and mid-lumbar discs the posterior fibres, when viewed in saggital section, pursue a vertical course, but this becomes progressively more curved convex posteriorly, developing a sharp hairpin bend in the lowest intervertebral disc. This posterior bulge is in just the site where the only pain fibres associated with the disc are situated and could therefore lead to backache. In the developing spine the lumbosacral curves become evident only as the child begins to stand and walk (Arey, 1965; De Palma and Rothman, 1970). It is man's upright posture which is responsible for the wedge shape of the L5/S1 disc and for the posterior bulging of the annulus fibrosus. Are we looking for some mysterious pathology causing back pain, when excessive bulging alone might be sufficient to account for this common spinal problem?

Nachemson (1966) measured the pressures within the nucleus pulposus in life and during normal spinal movements and calculated the tangential stresses produced within the annulus fibrosus. Under load most force is transmitted by the nucleus pulposus, but with disc degeneration this falls so that there must be an increase in the vertical stress on the annulus 


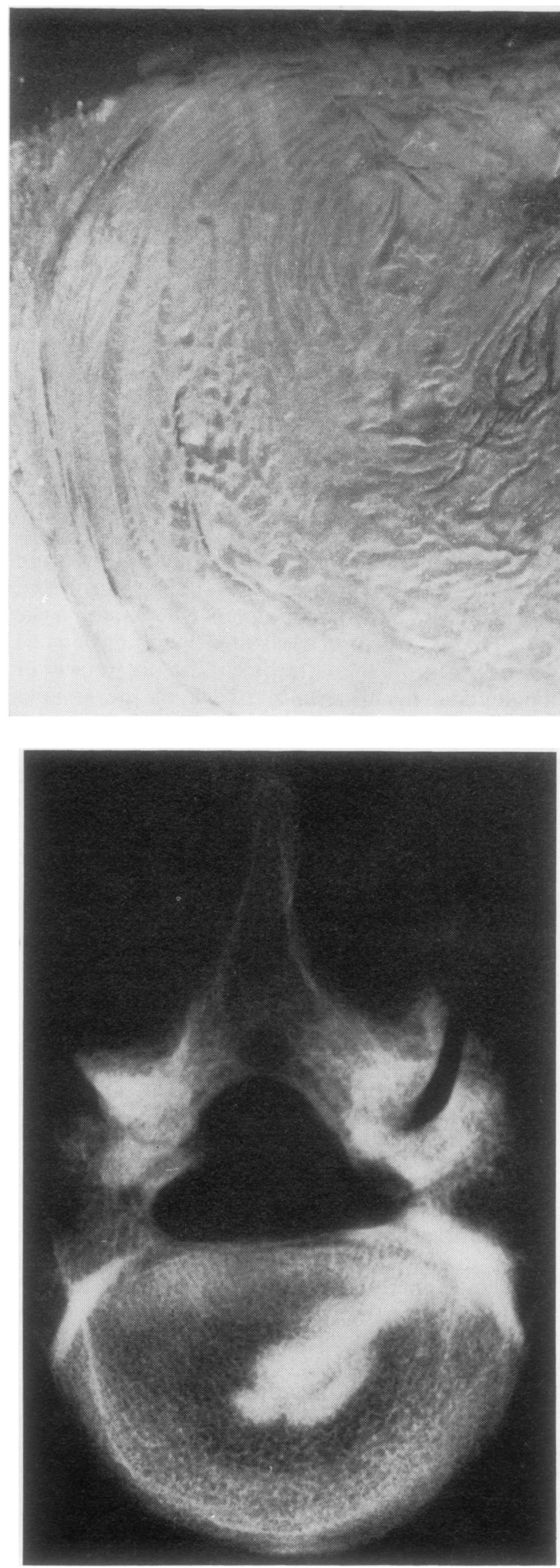

FIG. $7 \quad L_{5} S_{1}$ horizontal section. The disc is degenerate. It appears as if this degeneration has followed a central posterior hernia

FIG. 8 Axial discogram showing postero-lateral hernia

fibrosus. These studies were, however, restricted to the upper and mid-lumber discs. Disc prolapse producing nerve root damage usually affects the lowest lumbar discs and, in view of the wedge shape, the reported calculations may well not be relevant to the clinical problem. Moreover, Nachemson's calculations did not take account of the posterior bulge of the annulus fibrosus of the L5/S1 disc.

In a number of specimens, we observed central posterior herniation of the nuclear material through a narrow neck to lie beneath the posterior longitudinal ligament. This central hernia is again in the only area where pain fibres are in direct association with the disc. On plain radiography these lumbar discs appeared completely normal despite gross pathological changes. Can this be another mechanism for the production of undiagnosable spinal pain? The lowest lumbar disc shown in Figs $5 e$ and 7 showed marked degenerative changes which could well be a late result of posterior prolapse and loss of nuclear substance. Perhaps this central posterior herniation is a frequent feature which cannot be diagnosed in life yet produces back symptoms and leads to disc degeneration. Friberg (1948) called attention to posterior degeneration occurring in the lower lumbar discs and suggested that it might be due to the 
posterior concavity of the spine and the upright posture of man.

The shape of the nucleus pulposus is also of interest. In otherwise completely normal discs, postero-lateral extensions were sometimes observed. They often occurred at several levels in the same spine. Postero-lateral prolapse producing injury to nerve roots occurs in this direction and some individuals may be predisposed towards this type of disc damage.

The types of disc prolapse are well known (Schmorl and Junghanns, 1959) and central posterior herniation was described more than a century ago by Luschka (1858). The systemic nature of the lesions with one type of disc damage occurring at multiple levels in the same spine has not been appreciated. A localized weakness of disc structure could be responsible. This might be present in all discs, perhaps as an inherited characteristic. Stress and trauma alone could not account for these lesions. Evans and Lissner (1954), Brown, Hansen, and Yorra (1957), Roaf (1960), and Smith (1969) all showed that stress will fracture the vertebral endplates, with herniation of the nucleus into the vertebral bodies, rather than damage the normal annulus fibrosus.

In the presence of minimal radiological degenerative changes or none, gross abnormalities in disc structure were found, indicating that $x$ ray changes are relatively late manifestations of disc degeneration.
A normal spinal $x$ ray does not exclude disorders of the intervertebral discs.

\section{Summary}

The structure of the intervertebral discs has been studied by a variety of techniques. The nucleus pulposus is central in the upper lumbar discs but is often more posterior in the lower lumbar discs, and the posterior fibres of the annulus fibrosus then pursue a hairpin course at the posterior edge of the disc, producing a posterior bulge. This area is the only site in which there are pain fibres in association with the disc and this posterior bulging may well be one mechanism for the production of spinal pain. The normal nucleus pulposus often has posterolateral extensions which could predispose to posterolateral prolapse. Central posterior-herniation and postero-lateral herniation were observed at multiple levels in the same spines, indicating systemic abnormalities in disc structure. These changes were observed despite normal plain spine $x$ rays. This $x$ ray is of no value in assessing disc structure.

This work was performed with the aid of grants from the Arthritis and Rheumatism Council and the Association of Friends of the Royal National Hospital for Rheumatic Diseases, Bath. M.I.V.J. is in receipt of a research grant from the Medical Research Council. We wish to thank Mr. Dennis Hayward for his help.

\section{References}

AREY, L. B. (1965) "Developmental Anatomy." Saunders, Philadelphia and London.

Brown, T., Hansen, R. J., AND Yorra, A. J. (1957) J. Bone Jt Surg., 39A, 1135 (Some mechanical tests on the lumbosacral spine with particular reference to the intervertebral discs)

De Palma, A. F., AND Rothman, R. H. (1970) “The Intervertebral Disc.' Saunders, Philadephia, London and Toronto

Evans, F. G., AND LissNer, H. R. (1954) J. Bone Jt Surg. 36A, 185 (Strength of intervertebral discs)

FRIBERG, S. (1948) Acta orthop. scand., 17, 224 (Anatomical studies of lumbar disc degeneration)

HirSCH, C., IngelmarK, B.-E., AND Miller, M. (1963) Ibid., 33, 1 (The anatomical basis for low back pain)

Jackson, H. C., Winkelmann, R. K., AND Bickel, W. H. (1966) J. Bone Jt Surg., 48A, 1272 (Nerve endings in the human lumbar spinal column and related structures)

LAWRENCE, J. S. (1969) Ann. rheum. Dis., 28, 121 (Disc degeneration. Its frequency and relationship to symptoms)

LuSCHKA, H. vON (1858) 'Die Halbgelenke des Menschlichen Körpers.' Reimer, Berlin

NACHEMSON, A. (1966) Clin. orthop., 45, 107 (The load on lumbar disks in different positions of the body)

Pedersen, H. E., Blunck, C. F. J., AND Gardner, E. (1956) J. Bone Jt Surg., 38A, 377 (An anatomy of lumbosacral posterior rami and meningeal branches of spinal nerves (sinu-vertebral nerves)

RoAF, R. (1960) Ibid., 42B, 810 (A study of the mechanics of spinal injuries)

Roofe, P. G. (1940) Arch. Neurol. Psychiat., 44, 100 (Innervation of annulus fibrosus and posterior longitudinal ligament)

Schmorl, G., AND Junghanns, H. (1959) 'The Human Spine in Health and Disease,' trans. S. P. Wilk and L. S. Goin. Grune and Stratton, New York and London

SмIтH, F. P. (1969) J. Neurosurg., 30, 134 (Experimental biomechanics of intervertebral disc rupture through a vertebral body)

StILwell, D. L. (1956) Anat. Rec., 125, 139 (The nerve supply of the vertebral column and its associated structures in the monkey)

WYKE, B. (1970) Rheum. Phys. Med., 10, 356 (The neurological basis of thoracic spinal pain) 